\title{
Is quantum field theory a genuine quantum theory? Foundational insights on particles and strings
}

\author{
Hrvoje Nikolić \\ Theoretical Physics Division, Rudjer Bošković Institute, \\ P.O.B. 180, HR-10002 Zagreb, Croatia \\ hrvoje@thphys.irb.hr
}

November 9, 2018

\begin{abstract}
Practically measurable quantities resulting from quantum field theory are not described by hermitian operators, contradicting one of the cornerstone axioms of orthodox quantum theory. This could be a sign that some of the axioms of orthodox quantum theory should be reformulated. A non-orthodox reformulation of quantum theory based on integral curves of particle currents is advocated and possible measurable manifestations are discussed. The consistency with particle creation and destruction requires particles to be extended objects, which can be viewed as a new motivation for introducing string theory. Within this reformulation, an indirect low-energy test of string theory is also possible.
\end{abstract}

PACS numbers: 03.70.+k, 03.65.Ta, 11.25.-w

Keywords: Quantum field theory; particles; strings.

\section{Introduction}

When quantum field theory (QFT) is compared to the so-called first quantization of nonrelativistic point particles, it is frequently claimed that QFT is nothing but the standard rules of first quantization applied to different classical degrees of freedom - fields. Yet, such a claim is not completely true. For example, fermionic fields do not really have a classical counterpart and do not represent quantum observables. Hermitian operators representing quantum observables can be constructed by taking products of fields in which the number of fermionic fields is even. An example is the energy-momentum tensor $\hat{T}_{\mu \nu}(x)$. Nevertheless, although such operators correspond to quantities that should be measurable in principle, they are not quantities measured in practice. Instead, practically measurable predictions resulting from QFT 
are properties of particles. On the other hand, the theoretical concept of particles in interacting QFT is rather artificial (see, e.g., [1] and references therein).

One measurable property of particles is their number, which can be described by the particle number operator $\hat{N}$ constructed from fields. Still, the number of particles is not the only property of particles that we measure. Instead, we also measure some additional properties of particular particles, such as their momenta and positions. The momentum operator is not a problem; it can be defined in QFT, which allows also to reproduce the predictions of single-particle QM in the momentum space. The problem is the position operator. QFT does not contain a position operator. Single-particle relativistic QM does not contain it either (see, e.g., [1] and references therein). On the other hand, particle detectors in high-energy physics measure particle positions. In most practical situations this is not really a problem, because particle-physics phenomenologists have developed successfull intuitive rules that allow to predict the distribution of particle positions from scattering amplitudes in the momentum space. Still, there is a problem as a matter of principle: If the position operator is not really defined in QFT, while the position of particles is a quantity that we really measure when compare the predictions of QFT with experiments, can we really say that QFT is a genuine quantum theory?

One possible response to this problem is that the position is just a classical label, similar to time in nonrelativistic QM. Such an interpretation is indeed a natural one if one views field operators $\hat{\phi}(\mathbf{x}, t) \equiv \hat{\phi}(x)$ as the fundamental objects from which everything else should be derived. However, from such a theoretically natural way of viewing QFT, there is no way to derive the experimentally confirmed QM rule that the nonrelativistic wave function $\psi(\mathbf{x}, t)$ defines quantum probabilities $|\psi(\mathbf{x}, t)|^{2}$ of particle positions. Are nonrelativistic QM and QFT two independent theories describing different objects? If so, then why, in many situations, both theories describe the same particles? If, on the other hand, nonrelativistic QM is to be derived from more fundamental QFT, then how to derive the probabilistic interpretation of nonrelativistic $\psi(\mathbf{x}, t)$ by starting from the axioms of QFT? It seems that the standard orthodox formulation of quantum theory cannot answer these questions. Therefore, the purpose of this paper is to reformulate some basic principles of quantum theory, with the intention to obtain a self-consistent theory the axioms of which are compatible with all existing observations. As a byproduct, we also find that such a reformulated quantum theory leads to some testable predictions that cannot be obtained with the conventional orthodox formulation.

\section{The roles of particles and fields}

Our starting point is the fact that all measurements confirming the predictions of QFT are actually measurements of the properties of particles. Therefore, particles play the central role in our reformulation of quantum theory. Still, the success of QFT cannot be denied, so quantum fields play some role as well, but not as fundamental directly measurable quantities, but as auxiliary mathematical objects from which the properties of physical particles are calculated. (From such a point of view, the expres- 
sion "second quantization" seems more suitable than the expression "quantum field theory".) In the simplest case of spinless uncharged particles, the relation between quantum fields and particles is encompassed by the equalities of the form $[2,3]$

$$
\psi\left(x_{1}, \ldots, x_{n}\right)=S_{\left\{x_{a}\right\}}\left\langle 0\left|\hat{\phi}\left(x_{1}\right) \cdots \hat{\phi}\left(x_{n}\right)\right| \Phi\right\rangle
$$

where $\psi$ is the (unnormalized) $n$-particle wave function, $|\Phi\rangle$ is the QFT state in the Heisenberg picture (in general, $|\Phi\rangle$ is a superposition of states with different numbers of particles $n),|0\rangle$ is the vacuum, and $S_{\left\{x_{a}\right\}}$ denotes the symmetrization over all $x_{a}, a=1, \ldots, n$, needed because the field operators do not commute for nonequal times. The expression (1) can be easily generalized to other types of particles as well, including the fermionic ones [4]. The expression (1) is valid even in the case of interacting fields $[3,4]$, but, for simplicity, we put emphasis on the free case.

When the field satisfies the Klein-Gordon equation $\left(\partial^{\mu} \partial_{\mu}+m^{2}\right) \hat{\phi}(x)=0$, then $\psi$ satisfies $n$ Klein-Gordon equations

$$
\left(\partial_{a}^{\mu} \partial_{a \mu}+m^{2}\right) \psi\left(x_{1}, \ldots, x_{n}\right)=0
$$

one for each $a$. Consequently, the quantity $|\psi|^{2}$ cannot be interpreted as a probability density, because such probability would not be conserved. Instead, one can construct $n$ conserved Klein-Gordon currents

$$
j_{a \mu}=i \psi^{*} \stackrel{\leftrightarrow}{\partial}_{a \mu} \psi, \quad \partial_{a \mu} j_{a}^{\mu}=0,
$$

but the time components $j_{a 0}$ cannot be interpreted as probability densities either, as they can be negative. (Note also that such currents are related to a hermitian uncharged field, so an interpretation in terms of charge currents would also be inappropriate.) Still, in the nonrelativistic limit, one should recover the standard rule that $|\psi|^{2}$ represents the probability density. This suggests that the probabilistic interpretation of $\psi$ should be emergent, rather than fundamental. This motivates us to search for a fundamentally deterministic interpretation of $\psi$, from which, in the nonrelativistic limit, the usual probabilistic interpretation can be derived as an effective theory.

\section{Bohmian interpretation}

Fortunately, in the nonrelativistic limit, the simplest way to achieve this is known for more than 50 years: the Bohmian interpretation of nonrelativistic QM $[5,6,7]$ provides a fundamentally deterministic explanation of all probabilistic predictions of orthodox QM, as long as the predictions refer to measurements of observables described by hermitian operators. Nevertheless, the Bohmian interpretation is technically more complicated than the orthodox fundamentally probabilistic interpretation, which is the main reason that it is usually ignored by physicists, despite the fact that it may have practical applications as well [8]. In this paper, however, the motivation for introduction of the Bohmian interpretation is significantly extended. Our goal is not only to reproduce the predictions of nonrelativistic QM, but also to extend the 
theory to the relativistic regime. To repeat, in this regime the axioms of orthodox QM are not appropriate, as there is no hermitian operator corresponding to the measured particle positions.

For the case in which the particle creation and destruction is neglected, the appropriate relativistic generalization of the Bohmian interpretation is suggested in [9] and further developed in [10]. The crucial role is played by the integral curves (see also [11]) of the vector field (3). The integral curves can be represented by functions $X_{a}^{\mu}(\tau)$ satisfying

$$
\frac{d X_{a}^{\mu}(\tau)}{d \tau}=j_{a}^{\mu}
$$

where $\tau$ is an auxiliary affine parameter along the integral curves. The functions $X_{a}^{\mu}(\tau)$ can be viewed either as one curve in the $4 n$-dimensional configuration space, or $n$ synchronized curves in 4-dimensional spacetime. This makes Bohmian nonlocality consistent with the principle of relativistic covariance, so that a "preferred" synchronization between different particles is chosen dynamically, essentially by the choice of initial conditions (see also [12]). As the currents (3) may be spacelike at some regions of spacetime, superluminal particle velocities and motions backwards in time are also possible. Nevertheless, when particle velocities or positions are measured appropriately, measured particle velocities can only correspond to non-superluminal eigenvalues of the velocity operator, while multiple positions of a single particle at a single time cannot be observed [3, 10]. Still, as we discuss below, some nontrivial measurable manifestations of superluminal velocities and motions backwards in time are possible.

\section{Measurable predictions}

As demonstrated in [10], in the relativistic case, the measurable probabilistic predictions emerging from (4) may differ from more conventional interpretations that do not involve a calculation of integral curves of the particle current. To discuss it, we consider the case of a single particle with the current $j^{\mu}$. In general, since we deal with a fundamentally deterministic theory, there is a priori no simple mathematical rule that gives us a probability density of particle positions. Thus, the fact that $j^{0}$ cannot be interpreted as a probability density does not represent a fundamental problem. Nevertheless, in the nonrelativistic approximation in which $j^{0} \propto|\psi|^{2}$, the usual quantum probability distribution $|\psi|^{2}$ emerges as a distribution that corresponds to the quantum equilibrium $[13,14]$. In general, if one knows the distribution at some particular initial time, one can find the distribution at any other time by calculating the relativistic particle trajectories for all possible initial positions of the particles. Thus, in the fully relativistic case, one finds a nontrivial measurable prediction when the interaction with the apparatus that measures particle positions starts at some particular time $t_{1}$, provided that $j_{0}<0$ at some parts of the $t_{1}$-hypersurface. Let us briefly explain it. (For more details we refer the reader to [10].) Negative $j_{0}$ corresponds to a particle moving backwards in time. However, since the wave function (1) contains only positive frequencies, the motion backwards in time is only a transient 
phenomenon for a given particle trajectory. Thus, in order for a particle to move backwards in time at $t_{1}$, it must come to $t_{1}$ from larger times, by changing the direction of motion from a motion forward in time to a motion backwards in time. Clearly, this change of the direction of motion must happen at some time $t>t_{1}$. However, since the 4-velocity on a given spacetime point is unique, such a motion must also involve a motion is space, not only in time. On the other hand, a typical measuring apparatus that measures a particle position does not allow a motion in space $[5,6,7]$. Consequently, since the measuring apparatus is turned on for $t>t_{1}$, the effect of measurement is that the particle cannot arrive at points on the $t_{1}$-hypersurface at which $j_{0}$ is negative. Thus, one finds the probability density [10]

$$
\rho\left(\mathbf{x}, t_{1}\right)= \begin{cases}j_{0}\left(\mathbf{x}, t_{1}\right) & \text { on } \Sigma^{\prime}, \\ 0 & \text { on } \Sigma^{+} \cup \Sigma^{-},\end{cases}
$$

where $\Sigma^{-}$is the part of the $t_{1}$-hypersurface at which $j_{0}<0, \Sigma^{+}$is the part of the $t_{1^{-}}$ hypersurface that is connected to $\Sigma^{-}$by Bohmian trajectories for which $t<t_{1}$, and $\Sigma^{\prime}$ represents all other points of the $t_{1}$-hypersurface. We emphasize that this measurable result cannot be obtained without calculating the trajectories. The most surprising feature of this prediction is that the particle cannot be found at some positions at which the wave function does not vanish. This could be observed experimentally for a state described by a coherent superposition of two significantly different frequencies $\omega_{1}$ and $\omega_{2}$. Unfortunately, such an experiment requires very fast switching (lasting much shorter than $\left|\omega_{1}-\omega_{2}\right|^{-1}$ ) of the measuring apparatus at $t_{1}$, which turns out to be difficult to achieve with the present-day technology, given that the frequencies are sufficiently different to allow for a measurable effect [15]. Nevertheless, different (hopefully more feasible) measurable manifestations based on explicit calculations of particle trajectories are also conceivable [16].

\section{Particle creation and destruction and the role of strings}

One of the appealing features of the Bohmian interpretation of quantum particles is the fact that it avoids discontinuous jumps corresponding to the so-called wavefunction collapses. All evolution is fully continuous, described by the deterministic wave equation such as (2) and the attributed deterministic particle trajectories described by (4). However, the problem is to reconcile it with the fact that particles may also get created or destructed. One possibility is to introduce singular points at which particle trajectories begin or end [17]. Another possibility is to introduce an additional continuously changing property of particles called effectivity [3]. Still, both possibilities seem rather artificial. In addition, both possibilities require an explicit use of QFT, which seems unappealing for a theory that is fundamentally supposed to be a theory of particles, rather than fields. So, how to describe particle creation and destruction in a continuous way without postulating new quantities such as effectivities? Below we argue that the requirement of continuous creation and destruction of 
particles naturally leads to string theory. In this way, string theory can be viewed as a natural consequence (or even a prediction) of the Bohmian interpretation.

Consider a decay of one particle with the trajectory $X_{1}^{\mu}(\tau)$ into two new particles with the trajectories $X_{2}^{\mu}(\tau)$ and $X_{3}^{\mu}(\tau)$. All together, we have 3 trajectories $X_{a}^{\mu}(\tau)$, $a=1,2,3$. How to describe the transition from $X_{1}^{\mu}(\tau)$ to $X_{2}^{\mu}(\tau)$ and $X_{3}^{\mu}(\tau)$ in a continuous way without singular points at which these trajectories begin or end? The most obvious answer is by allowing the label $a$ to attain not only discrete integer values, but also real values that can continuously interpolate between the integer ones. In general, there may be $p$ independent real variables $\sigma_{1}, \ldots, \sigma_{p}$, so the trajectories $X_{a}^{\mu}(\tau)$ get replaced by more general functions $X^{\mu}\left(\sigma_{1}, \ldots, \sigma_{p}, \tau\right)$. Geometrically, such general functions represent $p$-dimensional extended objects evolving with "time" $\tau$. Further, if such objects are considered to be fundamental, one requires that their quantization does not lead to unsurmountable divergences, which excludes the cases $p>1$ [18]. This leads to functions $X^{\mu}(\sigma, \tau)$, which are nothing but strings in spacetime. Indeed, strings are already known to be a good candidate for a more fundamental theory that should replace the usual theory of particles and fields [18, 19]. In fact, it has already been argued that, if strings are assumed to be the fundamental objects, the Bohmian interpretation appears as a natural interpretation [20, 21, 22]. Our result above shows also the opposite: if the Bohmian interpretation is assumed, then strings appear as natural objects. Loosely speaking, strings are a prediction of the Bohmian interpretation.

In string theory, one can describe string creation and destruction without introducing an analog of fields. (String field theory is also a possible modification of string theory, but such a modification does not seem to be necessary and there are indications that it may not be the correct way to go [23].) The $n$-particle wave function $\psi\left(x_{1}, \ldots, x_{n}\right)$ generalizes to the bosonic string wave functional $\Psi[X(\sigma)] \equiv \Psi[X]$. Allowing functions $X^{\mu}(\sigma)$ to be discontinuous, the wave functional $\Psi[X]$ describes not only 1 -string states, but also $n$-string states, as well as amplitudes for processes in which the number of strings changes by string splitting [22]. There is no need for a stringy analog of (1). In the case of superstrings, the wave functional further generalizes to $\Psi[X, M]$, where $M(\sigma)$ are indices in the Hilbert space attributed to the operator corresponding to the anticommuting superstring coordinate $\psi^{\mu}(\sigma)[22]$. The particle current (3) generalizes to the superstring current [22]

$$
J_{\mu}[X ; \sigma)=i \int[d M] \Psi^{*}[X, M] \frac{\overleftrightarrow{\delta}}{\delta X^{\mu}(\sigma)} \Psi[X, M]
$$

Eq. (4) generalizes to

$$
\frac{\partial X^{\mu}(\sigma, \tau)}{\partial \tau}=J^{\mu}[X ; \sigma)
$$

which describes the continuous deterministic motion of strings, including the string splitting [22]. 


\section{Low-energy test of string theory}

To discuss possible measurable manifestations of our non-orthodox reformulation of string theory, it is convenient to study the implications on particles of the Standard Model. The main idea is to consider an appropriate low-energy string state $\Psi$ and its effective description in terms of the corresponding particle wave function $\psi$. Then from (6) we obtain a particle current generalizing (3) to higher spins, which leads to non-orthodox measurable predictions such as (5). For example, the photon wave function is obtained from a massless vector state of the open string, leading to a general 1-photon wave function

$$
\psi(x)=\sum_{k} \sum_{s} c_{s}(k) e^{-i k \cdot x}
$$

where $k^{\mu}$ is the particle momentum, $s$ labels $D-2$ independent polarization vectors $\zeta_{s}^{\alpha}(k)$ satisfying $k_{\alpha} \zeta_{s}^{\alpha}(k)=0$, and $c_{s}(k)$ are arbitrary coefficients corresponding to an arbitrary superposition of states with various momenta and polarizations. The corresponding low-energy 1-photon current resulting from (6) takes the same form as (3), that is

$$
j_{\mu}=i \psi^{*} \stackrel{\leftrightarrow}{\partial}_{\mu} \psi
$$

This should be contrasted with the 1-photon current that would naturally emerge directly from QFT [11]

$$
j_{\mu}^{\text {field }}=i \psi_{\alpha}^{*} \stackrel{\leftrightarrow}{\partial}_{\mu} \psi^{\alpha}
$$

where $\psi^{\alpha}$ is obtained from a spin-1 analog of (1), leading to

$$
\psi^{\alpha}(x)=\sum_{k} \sum_{s} c_{s}(k) \zeta_{s}^{\alpha}(k) e^{-i k \cdot x} .
$$

In general, the predictions from these two different 1-photon currents differ, provided that the superposition contains contributions from various non-collinear momenta $k$. In principle, this difference could be used for an indirect low-energy test of string theory.

A more dramatic difference between the string-based particle current and the fieldbased particle current occurs for spin- $\frac{1}{2}$ particles. The field-based 1-electron current is the Dirac current

$$
j_{\mu}^{\text {field }}=\psi^{\dagger} \gamma^{0} \gamma_{\mu} \psi
$$

where $\psi$ is the spinor with the c-number valued components $\psi_{m}$, while $\gamma_{\mu}$ are the Dirac matrices. The string-based low-energy 1-electron current resulting from (6) is

$$
j_{\mu}=i \psi^{\dagger} \stackrel{\leftrightarrow}{\partial}_{\mu} \psi
$$

where the index $m$ originates from $M[22]$. In the "field-frame" the current (13) does not seem to transform as a vector, but in the "string-frame" it is the Dirac current (12) that does not seem to transform as a vector. In [22] it is interpreted as an existence

of a preferred foliation of spacetime at the level of effective field theory. The most 
notable difference between these two currents is the fact that $j_{0}^{\text {field }}$ in (12) cannot be negative, so nontrivial probability distributions such as (5) cannot emerge from (12). Therefore, the experimental search for such nontrivial probability distributions of electrons would be an indirect low-energy test of string theory.

A more specific proposal for testing the difference between probabilistic predictions of (12) and (13) is discussed in detail in [24]. Let us briefly review it here. In 4 dimensions, the wave function of an ultrarelativistic electron with spin $+\frac{1}{2}$ having energy $\omega_{p}$ and moving in the $x$-direction is

$$
\varphi_{p}=\frac{1}{\sqrt{2}}\left(\begin{array}{l}
1 \\
0 \\
0 \\
1
\end{array}\right) e^{-i \omega_{p}(t-x)} .
$$

The main idea is to prepare the electrons in a superposition of two different equally probable frequencies $\omega_{1}$ and $\omega_{2}$. Thus, the wave function in (12) is

$$
\psi=\frac{1}{\sqrt{2}}\left(\varphi_{1}+\varphi_{2}\right)
$$

where $\varphi_{1}, \varphi_{2}$ denote spinors (14) with frequencies $\omega_{1}, \omega_{2}$, respectively. On the other hand, the Klein-Gordon-like current (13) requires a Klein-Gordon normalization of the wave function, so instead of (15), the corresponding wave function in (13) is

$$
\psi=\frac{1}{\sqrt{2}}\left(\frac{\varphi_{1}}{\sqrt{2 \omega_{1}}}+\frac{\varphi_{2}}{\sqrt{2 \omega_{2}}}\right) .
$$

Now we put the detector of electrons at a fixed position, say $x=0$. The probability density resulting from (12) with (15) is

$$
j_{0}^{\text {field }}(t)=1+\cos \left[(1-\eta) \omega_{1} t\right]
$$

where $\eta \equiv \omega_{2} / \omega_{1}$. Similarly, the probability density resulting from (13) with (16) is

$$
\left|j_{0}(t)\right|=\left|1+\frac{1+\eta}{2 \sqrt{\eta}} \cos \left[(1-\eta) \omega_{1} t\right]\right| .
$$

For $\omega_{1} \neq \omega_{2},(17)$ and (18) are different. The source of the difference lies in the fact that $j_{0}^{\text {field }}$ defined by (12) does not involve a time-derivative while $j_{0}$ defined by (13) does, which causes substantial differences for superpositions of different frequencies. As discussed in more detail in [24], the oscillatory patterns (17) and (18) can, at least in principle, be distinguished experimentally.

\section{Conclusion}

To conclude, our results suggest that quantum field theory is neither a quantum theory in the orthodox sense, nor a field theory in a naive sense. It is not an orthodox 
quantum theory because practically measurable quantities resulting from QFT are particle positions, which are not described by hermitian operators. It is not a field theory in a naive sense because the fundamental measurable quantities at low energies are particles rather than fields. The fact that a relativistic position operator does not exist lead us to argue that the usual quantum probabilities are emergent, while the fundamental dynamics is deterministic. This suggests a relativistic Bohmian-like formulation of quantum theory, which, in the relativistic regime, leads to testable predictions. To make the existence of Bohmian particle trajectories consistent with particle creation and destruction without leading to singular points at which trajectories begin or end, it is natural to generalize pointlike particles to extended objects. This leads to a new derivation of string theory based on the assumption of Bohmian mechanics, which allows testable low-energy predictions of string theory as well.

\section{Acknowledgements}

This work was supported by the Ministry of Science of the Republic of Croatia under Contract No. 098-0982930-2864.

\section{References}

[1] H. Nikolić, Found. Phys. 37, 1563 (2007).

[2] S. S. Schweber, An Introduction to Relativistic Quantum Field Theory (Harper \& Row, New York, 1961).

[3] H. Nikolić, Found. Phys. Lett. 17, 363 (2004).

[4] H. Nikolić, Found. Phys. Lett. 18, 123 (2005).

[5] D. Bohm, Phys. Rev. 85, 166, 180 (1952).

[6] D. Bohm and B. J. Hiley, Phys. Rep. 144, 323 (1987).

[7] P. R. Holland, The Quantum Theory of Motion (Cambridge University Press, Cambridge, 1993).

[8] C. L. Lopreore and R. E. Wyatt, Phys. Rev. Lett. 82, 5190 (1999).

[9] K. Berndl, D. Dürr, S. Goldstein and N. Zanghì, Phys. Rev. A53, 2062 (1996).

[10] H. Nikolić, Found. Phys. Lett. 18, 549 (2005).

[11] H. Nikolić, Int. J. Mod. Phys. A22, 6243 (2007).

[12] H. Nikolić, AIP Conf. Proc. 844, 272 (2006); quant-ph/0512065.

[13] A. Valentini, Phys. Lett. A156, 5 (1991). 
[14] D. Dürr, S. Goldstein and N. Zanghì, J. Stat. Phys. 67, 843 (1992).

[15] M. Genovese, private communication.

[16] H. Nikolić, Found. Phys. 38, 869 (2008).

[17] D. Dürr, S. Goldstein, R. Tumulka and N. Zanghì, Phys. Rev. Lett. 93, 090402 (2004).

[18] M. B. Green, J. H. Schwarz and E. Witten, Superstring Theory (Cambridge University Press, Cambridge, 1987).

[19] J. Polchinski, String Theory (Cambridge University Press, Cambridge, 1998).

[20] H. Nikolić, Eur. Phys. J. C47, 525 (2006).

[21] H. Nikolić, Eur. Phys. J. C50, 431 (2007).

[22] H. Nikolić, hep-th/0702060.

[23] J. Polchinski, hep-th/9411028.

[24] H. Nikolić, arXiv:0806.1431. 